\title{
A short-term load forecasting taking into account the correlation of integrated energy load
}

\author{
Xianjun Qi ${ }^{1,2}$, Qinghui Chen ${ }^{2 *}$ and Xiwei Zheng ${ }^{2}$ \\ ${ }^{1}$ Anhui Provincial Laboratory of Renewable Energy Utilization and Energy Saving, Hefei University of Technology, Hefei, Anhui \\ Province, 230009, China \\ ${ }^{2}$ School of Electrical and Automatic Engineering, Hefei University of Technology, Hefei, Anhui Province, 230009, China
}

\begin{abstract}
This paper proposes a short-term load forecasting method that takes into account the correlation of integrated energy load. The method use wavelet packet to decompose the electric cooling and heating load in frequency bands, analyze the cross-correlation of the electric cooling and heating load in each frequency band, and choose different forecasting methods according to the strength of the correlation to reflect the cross-correlation of the load itself; the method use recurrent neural network as a forecasting model to reflect the autocorrelation of the load itself. Compared with putting the electric cooling and heating load into the same recurrent neural network or back propagation neural network for forecasting, the method in this paper considers the autocorrelation of the electric cooling and heating load itself and the crosscorrelation of the electric cooling and heating load in different frequency bands. This method reduces the average absolute percentage error of the load forecasting.
\end{abstract}

\section{Introduction}

Energy and environmental issues are the hotspots of today's society, and they affect the sustainable development of mankind. The existing energy supply methods have many disadvantages such as low conversion efficiency, low concentration, high cost. This makes the energy problem the primary factor affecting sustainable development. The integrated energy system is a system that integrates electric, cooling and heating loads [1]. It is an important trend in energy development and plays an important role in promoting energy structure optimization, stimulating renewable energy consumption, and improving energy utilization.

Precise forecasting of integrated energy load is the basis for the design, operation and dispatch of integrated energy systems. In terms of integrated energy short-term load forecasting, statistical methods and intelligent algorithms are currently used. Literature [2] proposes an improved method based on Markov chain synthetic load neural network forecasting model. Literature [3] combines support vector machines and artificial neural networks to propose a combined forecasting method for forecasting cooling and heating loads.

The integrated energy electric, cooling and heating load is time series data, and its connected data points have strong autocorrelation. This autocorrelation should be fully considered when making forecast [4]. The forecast time of short-term load forecasting is generally from one day to one week. The general support vector machine model and back propagation neural network (BPNN) model can't take into account the autocorrelation between connected points in the forecast time. Recurrent neural network (RNN), as a memory network, is very suitable for solving time series problems. The biggest feature of RNN is that the output of a neuron at a certain moment can be re-input to the neuron at the next moment. This series network structure is suitable for processing time series data with autocorrelation at connected moments [5]. Therefore, this paper uses RNN as a basic forecasting model to deal with the autocorrelation of integrated energy load.

The difference between comprehensive energy load forecasting and single load forecasting is that there are multiple cross-correlated loads in the comprehensive energy system, and the cross-correlation performance is used to reduce the forecast error of the comprehensive energy load. Literature [6] uses Pearson coefficient to analyze the cross-correlation of various influencing factors, and selects appropriate factors as the input for comprehensive energy load forecasting. Literature [7] uses Copula theory to analyze the nonlinear crosscorrelation between multiple loads and between multiple loads and weather factors, and combines historical data of temperature, radiation intensity, humidity, electrical load, and cooling load to form a multiple load forecasting model. The input sample set. The abovementioned documents all carry out cross-correlation analysis on the comprehensive energy load in the time domain, but the regularity of the load in the time domain is poor, which is not conducive to reducing the forecast

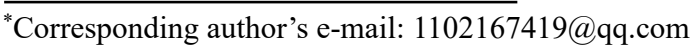


error. However, each load through wavelet packet decomposition (WPD) can show strong regularity and periodicity in each frequency band [8]. On this basis, the correlation analysis of the comprehensive energy load in each frequency band is carried out. Will help reduce forecast errors.

Based on the above analysis, this paper proposes a WPD-RNN forecasting method. The characteristics of this method are:

1) Analyse and utilize the cross-correlation of integrated energy load from the perspective of frequency domain. WPD, which helps reveal the detailed characteristics of the load, decomposes the integrated energy load into multiple frequency band components, and calculates the Pearson correlation coefficient between the integrated energy load components on each frequency band.

2) In each frequency band, analyse the crosscorrelation: the integrated energy load components with strong cross-correlation are put into the same RNN model for simultaneous forecasting to reflect the crosscorrelation between the loads; the integrated energy load components with weaker cross-correlation are placed in a separate RNN model for forecasting.

3) Finally, the forecasting results of each frequency band are added to obtain the electric, cooling and heating load forecasting results. It is verified by calculation examples that compared to putting the relevant integrated energy load into the same RNN model for forecasting or into the same BPNN model for forecasting, the WPDRNN forecasting method proposed in this paper can effectively reduce the integrated energy load forecast mean absolute percentage error (MAPE).

\section{WPD and cross-correlation analysis}

\subsection{WPD principle}

WPD is developed based on wavelet decomposition (WD). WPD can perform signal decomposition on the high and low frequency parts at the same time, and can adaptively select the corresponding frequency band to match the signal spectrum according to the signal characteristics and analysis requirements [9]. For fluctuating signals, using WPD can highlight the details of the signal. Therefore, this paper uses WPD to decompose the electric cooling and heating load data respectively.

Figure 1 is a three-layer wavelet packet decomposition structure diagram. In the figure, $S$ is the input signal. For integrated energy load forecasting, $S$ is the historical load data. The result of the decomposition is to finally map the signal $S$ into $2^{i}$ ( $i$ is the number of decomposition layers) wavelet packet subspaces [8].

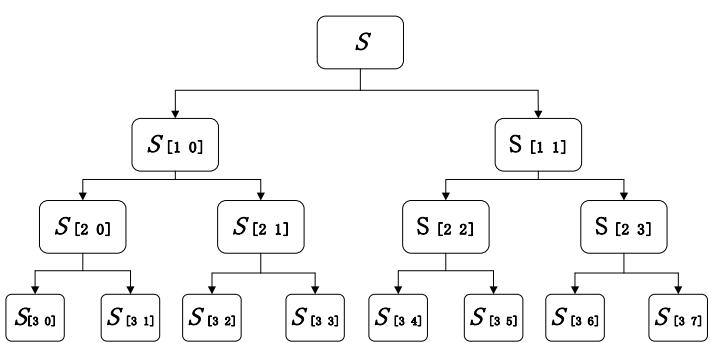

Fig.1 Structure diagram of three-layer wavelet packet decomposition

\subsection{Cross-correlation analysis}

The purpose of cross-correlation analysis in this paper is to perform cross-correlation analysis on the electric cooling and heating load components in each interval after WPD, and put the electric cooling and heating load replaced by cross-correlation into the same cyclic neural network model for forecasting. The influence of the consistent cross-correlation of electric cooling and heating load on the forecasting results. In this paper, Pearson correlation coefficient [10] is used to describe the cross-correlation of electric cooling and heating load.

The Pearson correlation coefficient is a quantitative indicator of the strength of the linear correlation between variables. Set the time series sum, and the correlation coefficient $\rho_{x y}$ is calculated as formula (1):

$$
\rho_{x y}=\frac{\sum_{i=1}^{N}\left(X_{i}-\bar{X}\right)\left(Y_{i}-\bar{Y}\right)}{\sqrt{\sum_{i=1}^{N}\left(X_{i}-\bar{X}\right)^{2}} \sqrt{\sum_{i=1}^{N}\left(Y_{i}-\bar{Y}\right)^{2}}}
$$

Where: $\bar{X}$ is the average of the time series $\left\{X_{i}\right\}$; $\bar{Y}$ is the average of the time series $\left\{Y_{i}\right\} ; N$ is the number of data in each time series. This paper takes $\left|\rho_{x y}\right|$ whether is greater than 0.2 as the evaluation criterion. If $\left|\rho_{x y}\right| \geq 0.2$, put the time series with cross correlation into the same model for forecasting; if $\left|\rho_{x y}\right| \leq 0.2$, forecast the time series and establish a model separately.

\section{RNN forecasting model}

The RNN structure diagram is shown in Figure 2. RNN includes input unit, output unit and hidden unit. The input set of the input unit is recorded as $\left\{x_{0}, x_{1}, \cdots, x_{t}, x_{t+1}, \cdots\right\}$; the output set of the output unit is recorded as $\left\{o_{0}, o_{1}, \cdots, o_{t}, o_{t+1}, \cdots\right\}$; and the output set of the hidden unit is recorded as $\left\{s_{0}, s_{1}, \cdots, s_{t}, s_{t+1}, \cdots\right\} . U, V$ and $W$ are the weight matrix from input unit to hidden unit, the weight matrix from hidden unit to output unit, and the weight matrix between hidden units[4]. 


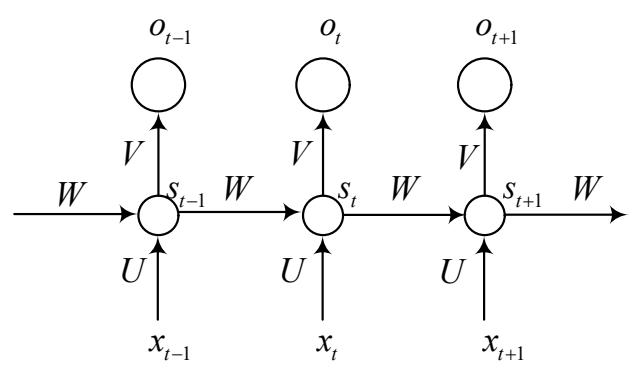

Fig.2 Structure of RNN

\section{WPD-RNN load forecasting method}

Use wavelet packet to decompose the pre-processed historical load data, calculate the correlation coefficient between the electric, cooling and heating load in each frequency band, construct different RNN forecasting models according to the correlation coefficient and perform load forecasting, and finally forecast the results of each frequency band Add up to get the result of electric cooling and heating load forecasting.

\section{Example analysis}

Take the integrated energy system of Arizona State University in the United States as an example. The integrated energy system of the campus consists of electric cooling and heating loads. The school's electric cooling and heating load data with a sampling interval of 1 hour in December 2018 was used to validate the method in this paper. The first 30 days of December 2018 were used as training samples for the establishment of the forecasting model. The last day (i.e. 2018 December 31) as a forecast sample to test the established forecast model.

Use the training data to train the above-built RNN models to obtain the parameters of the forecasting model, and then input the test data into the forecasting model to obtain the load forecasting value of each frequency band, and superimpose the final forecasting result. Figures 3, 4, and 5 are the comparison diagrams of the forecasted value and actual value of the electric cooling and heating load during the forecasting day. Figure 6 shows the relative error of the electric cooling and heating load within the forecast time.

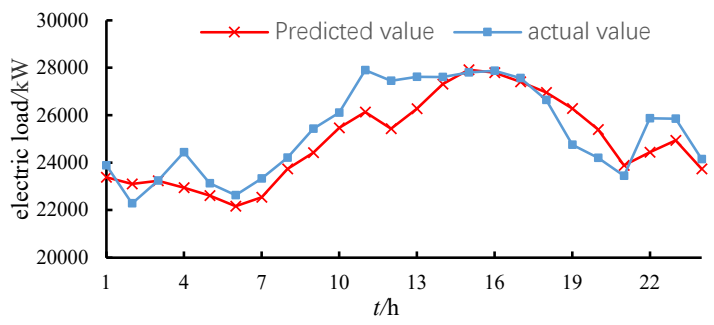

Fig.3 Results of electric load forecasting

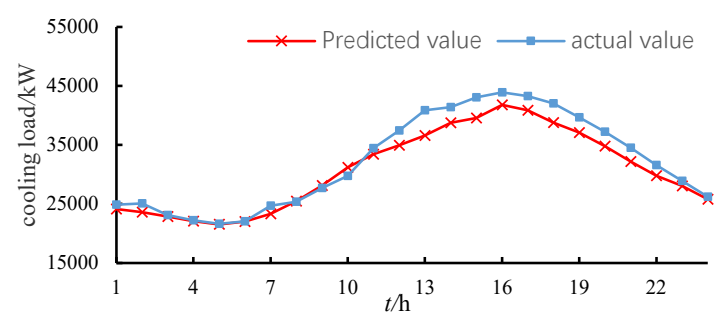

Fig.4 Results of cooling load forecasting

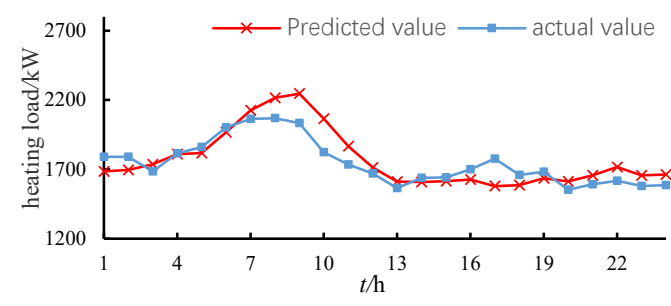

Fig.5 Results of heating load forecasting

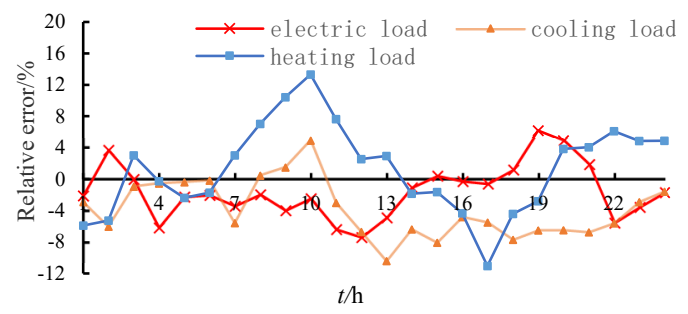

Fig.6 Relative error of electric cooling and heating loads forecasting

From Figures 3, 4, 5 and 6, it can be seen that the electric cooling and heating load forecasting curve obtained by the WPD-RNN forecasting method has a higher degree of fit with the actual electric cooling and heating load curve, which verifies the feasibility of the WPD-RNN forecasting method.

In order to further measure the effectiveness of the electric cooling and heating short-term load forecasting method proposed in this paper, the WPD-RNN forecasting method is compared with the RNN forecasting method and the BPNN forecasting method.

Figure 7 shows the MAPE of the forecasting results of the three forecasting methods. It can be seen that the forecasting method (WPD-RNN) proposed in this paper has the smallest MAPE.

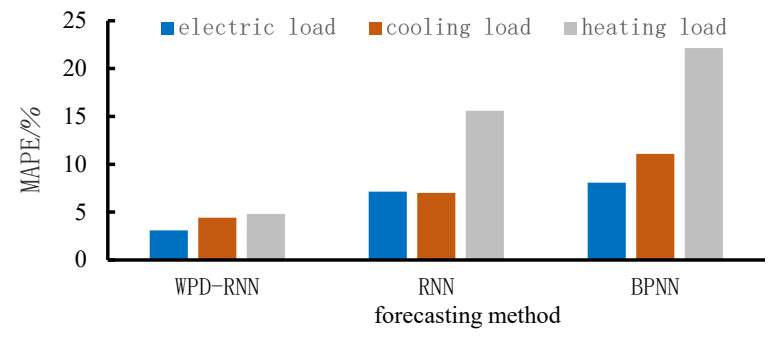

Fig.7 MAPE by three forecasting methods

Compared with the RNN prediction method, the MAPE of the electric cooling and heating load of the WPD-RNN prediction method is reduced by $4.06 \%$, $2.59 \%$, and $10.76 \%$ respectively. Compared with the 
BPNN prediction method, the electric cooling and heating load MAPE in the WPD-RNN prediction method decreases more significantly, which are $4.99 \%, 6.67 \%$ and $17.33 \%$ respectively. This is because the WPD-RNN forecasting method not only considers the crosscorrelation of the electric cooling and heating load in each frequency band, but also uses the memory function of RNN to reflect the autocorrelation of the electric cooling and heating load in the time series, so that the electric cooling and heating load The MAPE is lower.

In summary, compared with RNN and BPNN prediction methods, the WPD-RNN prediction method proposed in this paper fully considers the autocorrelation of electric cooling and heating load in time series and the cross-correlation in each frequency band, which can effectively reduce MAPE for comprehensive energy load forecasting.

\section{Conclusion}

The WPD-RNN forecasting method proposed in this paper is based on the RNN forecasting method, using WPD to obtain the load components of the electric cooling and heating load in different frequency bands, and then analyze the cross-correlation of the electric cooling and heating load on each frequency band. The method in this paper finally establishes the corresponding RNN model to predict each frequency band according to the analysis result. Through the research of this article, we can draw the following conclusions :

1) The memory function of RNN embodies the autocorrelation of comprehensive energy load, which helps to reduce the MAPE of load forecasting.

2) Using WPD to analyze the cross-correlation of the comprehensive energy load in each part provides a new idea for the cross-correlation analysis of the comprehensive energy load, which can further reduce the MAPE of load forecasting.

3) Comprehensively consider the gradual autocorrelation of the comprehensive energy load and the cross-correlation in all aspects, and establish a WPDRNN prediction model, which can give full play to the respective advantages of WPD and RNN, thereby effectively reducing the MAPE of load forecasting.

\section{Acknowledgments}

This work was supported by Institute of Economy and Te chnology, State Grid Anhui Electric Power Co., Ltd. (N o. B3440818K005)

\section{References}

1. Yu X., Xu X., Chen S. (2016) A brief review to integrated energy system and energy internet. Transactions of China Electro technical Society, 31: 1-13.

2. Zhang T., Li H., Hui Q. (2019) Integrated load forecasting model of multi-energy system based on markov chain improved neural network. In: Proceedings of the 11th International Conference on Measuring Technology and Mechatronics Automation. Thailand. pp. 454-457.

3. Kody M., Akshay S., Wesley J. (2014) Heating cooling and electrical load forecasting for a largescale district energy system. Energy, 74: 877-885.

4. Jin L., Li S., Hu B. (2020) RNN models for dynamic matrix inversion: a control-theoretical perspective. IEEE Transactions on Industrial Informatics, 14: 189-199.

5. Shi H., Xu M., Li R. (2018) Deep learning for household load forecasting-a novel pooling deep RNN. IEEE Transactions on Smart Grid, 9: 52715280.

6. Li S., Qi J., Bai X. (2019) A short-term load prediction of integrated energy system based on IPSO-WNN. Electrical Measurement and Instrumentation, 11: 1-7.

7. Ma J., Gong W., Zhang Z. (2019) Multiple load short-term prediction model of regional integrated energy system based on Copula theory and KPCA-GRNN. Advanced Technology of Electrical Engineering and Energy, 16: 1-7.

8. Ye R., Guo Z., Liu R. (2017) Wind speed and wind power forecasting method based on wavelet packet decomposition and improved Elman neural network. Transactions of China Electrotechnical Society, 32: 103-111.

9. Li J., Yang W. (2004) Ten lectures on wavelets. National Defense Industry Press, Beijing.

10. Chen N., Qian Z., Meng X. (2013) Multi-step ahead wind speed forecasting model based on spatial correlation and support vector machine. Transactions of China Electrotechnical Society, 28: $15-21$. 